\title{
Desafíos y consideraciones bioéticas de la investigación para la salud en colaboración entre países del Norte y del Sur*
}

\author{
Fernando José Andrade-Narvaez ${ }^{a}$
}

\begin{abstract}
Resumen: La investigación para la salud es esencial para enfrentar los retos actuales y futuros mediante la generación de nuevos conocimientos, que a su vez deben ser traducidos en mejores formas de prevenir y tratar las enfermedades, todo con el fin de lograr un desarrollo humano global sostenible. La tan necesaria investigación colaborativa Norte-Sur para la salud ha ido en franco aumento en las últimas décadas como respuesta a lo anterior. Por diversas razones, en esta interacción han surgido desafíos y cuestionamientos bioéticos que deben ser afrontados. En el presente trabajo se identifican 1) la asimetría; 2) el colonialismo; 3) la explotación; 4) la información, y 5) los comités de ética en investigación como los principales desafíos y se revisan los aspectos bioéticos que son necesarios atender. Resulta evidente la urgencia de construir una bioética de la investigación para la salud en colaboración entre países del Norte y países del Sur.
\end{abstract}

Palabras clave: bioética; salud; investigación; cooperación internacional

Recibido: 08 de enero de 2020 Aceptado: 25 de marzo de 2020

Disponible en línea: 15 de septiembre de 2020.

Cómo citar: Andrade-Narvaez FJ. Desafíos y consideraciones bioéticas de la investigación para la salud en colaboración entre países del Norte y del Sur. Rev. latinoam. bioet [Internet]. 15 de septiembre de 2020; 20(1). Disponible en: https://doi.org/10.18359/rlbi.4474

* Artículo de revisión

a Doctorado en Ciencias (Inmunología), Magister en Bioética, Especialidad en Medicina Interna, Licenciatura en Medicina. Profesor Investigador Titular "C" TC, Laboratorio de Inmunología, Centro de Investigaciones Regionales "Dr. Hideyo Noguchi", Universidad Autónoma de Yucatán, Mérida, Yucatán. Correo electrónico: anarvaez@correo.uady.mx ORCID • 


\title{
Bioethical challenges and considerations of research for health in collaboration amongst Southern and Northern Countries
}

\begin{abstract}
Health research is essential to face current and future challenges by generating new knowledge, which in turn must be translated into better ways to prevent and treat diseases; all of this in order to achieve sustainable global human development. The much-needed North-South collaborative research for health has been on the rise in recent decades in response to the above. Due to various reasons, bioethical challenges and questions that must be addressed have arisen in this interaction. In this work we have identified: 1) asymmetry, 2) colonialism, 3) exploitation, 4) information , and 5) the research ethics committees, as the main challenges. Additionally, the bioethical aspects to be addressed have been reviewed. The urgency to build the bioethics of health research in cooperation between Northern and Sothern countries becomes evident.
\end{abstract}

Keywords: bioethics; health; research; international cooperation

\section{Desafios e considerações bioéticas da pesquisa em saúde em colaboração entre países do Norte e do Sul}

Resumo: A pesquisa em saúde é essencial para enfrentar os desafios atuais e futuros mediante a geração de novos conhecimentos que, por sua vez, devem ter traduzidos em melhores formas de prevenir e tratar as doenças, a fim de atingir um desenvolvimento humano global duradouro. A tão necessária pesquisa colaborativa Norte-Sul em saúde tem aumentado nas últimas décadas como resposta a tudo isso. Por diversas razões, nessa interação vêm surgindo desafios e questionamentos bioéticos que devem ser enfrentados. Neste trabalho são identificados como os principais: 1) assimetria; 2) colonialismo; 3) exploração; 4) informação e 5) comitês de ética em pesquisa; além disso, são verificados os aspectos bioéticos que são necessários atender. É evidente a urgência de construir uma bioética da pesquisa em saúde em colaboração entre países do Norte e do Sul.

Palavras-chave: bioética; saúde; pesquisa; cooperação internacional 
-Ya veo-dijo la descendiente de Noé.

$$
\text { Sí-respondió el pájaro-.... }
$$

Por qué está tan claro como el día y la noche:

los bosques están siendo arrasados; las aguas envenenadas;

el aire se está oscureciendo y volviendo tóxico.

Y los gobiernos presuntuosos continúan proyectando su poder con éxito: para conmocionar y asombrar, masacrar, explotar y despojar.

Es cierto, no se puede salvar el mundo. ¿y si actuamos como si pudiera salvarse? Pues entonces... -Ya veo-repitió la descendiente de Noé. -Sí-dijo el pájaro agorero más animado-. Casi es posible que se pueda salvar el mundo.

\section{Introducción}

En primer lugar, es necesario precisar a qué nos referimos con los términos Norte y Sur, utilizados en las publicaciones oficiales de las Naciones Unidas y del Banco Mundial. Con el primer vocablo designamos a los países desarrollados, de altos ingresos o ricos, de Norte América, Europa, Japón, Australia y Nueva Zelanda. El segundo lo usamos para referirnos a los países en desarrollo, de bajos o medianos ingresos o pobres, de Latinoamérica, África y Asia (1).

El interés por la investigación colaborativa (collaborative research) entre países del Norte y del Sur emergió en 1983 al fundarse la denominada The Third World Academy of Sciences (2). Esta iniciativa se creó en el marco de la cooperación internacional con la intención de lograr que en los entonces llamados "países del tercer mundo" llegara a existir un avance en investigación y conocimientos científicos con respecto a los "países del primer mundo". Se consideró que el término tercer mundo estigmatizaba y era discriminatorio, por lo que esta terminología fue eliminada y se cambió el nombre a The World Academy of Sciences (TWAS). La relevancia de esta iniciativa radica en que por primera vez los científicos del mundo tomaron clara consciencia de que, ante problemas globales tales como el crecimiento poblacional, la destrucción del medio ambiente y el calentamiento global, con sus respectivas y reconocidas consecuencias negativas, era muy necesario trabajar en forma conjunta por y para un fin compartido: un desarrollo humano global sostenible respetando el medio ambiente.

Debe subrayarse que han transcurrido más de tres décadas, y podemos afirmar que la situación mundial no solamente no ha mejorado, sino que el daño del medio ambiente se ha agravado, faltando aún mucho por hacer para revertir o aminorar la grave desigualdad existente entre los países del Norte y los del Sur. En particular, la desigualdad existente en lo referente al estado de salud, la atención de la salud, la investigación en y para la salud 
y las políticas en salud es ampliamente reconocida, aun cuando la investigación para la salud se reconoce como un factor esencial para el desarrollo y es sin lugar a dudas uno de los medios para disminuir la abismal diferencia existente entre los países del Norte y del Sur (3).

La bioética analiza argumentativa e interdisciplinariamente situaciones dilemáticas valorativas surgidas en y desde las ciencias de la vida y de la salud debido a la creciente tecnificación que impacta la vida en toda su amplitud y a la variedad de paradigmas de valores existentes. Dicho análisis busca, con responsabilidad solidaria, llegar a consensos en los que prime el respeto por la dignidad humana y por la vida en general, tanto presente como venidera. La bioética, como tal, es una ética aplicada a un campo específico de la realidad (4).

La investigación colaborativa Norte-Sur para la salud presenta como característica sobresaliente su extrema complejidad debido a los múltiples factores subyacentes y determinantes involucrados, lo cual es en sí una razón esencial para que su realización conlleve un abordaje interdisciplinario. Esto a su vez plantea diversos cuestionamientos éticos que deben pensarse, y la bioética por su carácter interdisciplinario es sin lugar a dudas una vía idónea para realizar esta empresa.

Debido al ascendente interés por la investigación colaborativa Norte-Sur para la salud, o mejor dicho, al interés de los países del Norte en realizar investigaciones en los países del Sur, surge la necesidad de construir una cultura bioética que se aboque al análisis de los cuestionamientos éticos que surgen de una investigación en colaboración entre países de ambos hemisferios.

El presente trabajo se realizó con el fin de identificar los principales desafíos que la investigación colaborativa Norte-Sur para la salud ha enfrentado y que aún persisten. Es una revisión exhaustiva no de la literatura en inglés y español, sino del material bibliográfico trascendente y relevante con relación al problema de estudio. Además, este trabajo tampoco se enfocó en lo que ocurre en alguno de los países del Sur en particular para evitar cualquier sesgo. Así, en consecuencia, es primordial revisar las cuestiones bioéticas relacionadas con los desafíos que se identifiquen.

\section{Metodología}

Para alcanzar el propósito, se realizó una búsqueda bibliográfica de la literatura más relevante en los buscadores PubMed, Google Scholar, SciELO, Redylac, LILAC ES y BVs, utilizando palabras claves en inglés (bioethical issues; health research; international cooperation) y en español (consideraciones bioéticas; investigación para la salud; cooperación internacional). Llama la atención la abundante literatura en inglés en contraste con la existente en español.

\section{Resultados}

\section{Desafíos}

Si bien existe en el ámbito internacional un consenso con respecto a lo planteado anteriormente - la existencia de un abismo profundo entre los países del Norte y los del Sur-, es un hecho que para poder lograr un cambio se deben identificar los desafíos (retos, obstáculos, adversidades). Es importante recalcar que las diferencias persisten a pesar de las iniciativas de organismos internacionales, que han sido revisadas sin éxito por las mismas organizaciones (5). A continuación se detallan los desafíos que se han identificado en la revisión de la literatura consultada.

\section{Asimetría (disparidad, desigualdad)}

En un excelente y extenso trabajo, Gaillard se cuestionaba hace ya unos 25 años si la colaboración Norte-Sur era posible debido a la enorme asimetría, disparidad y desigualdad existente, la cual señaló como el principal reto para lograr una eficiente cooperación internacional (6).

Evidentemente, la investigación colaborativa para la salud entre países del Norte y del Sur ha enfrentado una situación de asimetría muy acentuada, que, con el paso de los años y a pesar de todos los intentos y esfuerzos, no se ha logrado revertir. Es importante subrayar también que, además de las enormes diferencias en cuanto a desarrollo, organización política y situación económica, existen diferencias culturales que dificultan en demasía las posibilidades de trabajar en forma conjunta. 
Maina-Ahlberg ET AL. (7) revisaron los retos de la investigación colaborativa Norte-Sur enfatizando en la complejidad de la interacción producto de la participación de países e instituciones en condiciones socioeconómicas muy diferentes, las cuales dificultan el logro de los objetivos propuestos. Se fundamentan y citan las restricciones y limitaciones identificadas por la Comisión de Investigación en Salud para el Desarrollo (Commission on Health Research for Development) puntualizando las escasas oportunidades de los investigadores de países de bajos ingresos para lograr capacitarse, la debilidad e inestabilidad del ambiente institucional donde laboran y los limitados fondos destinados al apoyo de la investigación en salud en dichos países (8).

Una de las características y diferencias más notorias es que los países del Sur son con frecuencia áreas endémicas de enfermedades tropicales desatendidas, que afectan a poblaciones igualmente desatendidas (9). Es bien sabido que en los países del Sur la carga por enfermedades tropicales desatendidas es muy alta debido en parte a que en ellos existen múltiples factores determinantes de la salud que se ven implicados en esta desatención.

Además de esta carga, es común que en los países del Sur exista deficiencia en la atención de la salud y en los recursos destinados para esta. Esta situación da lugar en la práctica a una relación de inequidad en la que los países del Norte son vistos y se comportan como "proveedores" y los del Sur como los "receptores", presentando un escenario de discriminación al considerar a los países ricos como "superiores" y, por tanto, a los países pobres como "inferiores" (10).

Es ampliamente reconocido el hecho de que en los países del Sur hay, con elevada frecuencia, un sin número de restricciones que afectan de manera general la capacidad de investigación científica; algunas de estas son: 1) asignación insuficiente de recursos financieros y materiales; 2) fallas por parte de los tomadores de decisión (políticos responsables de la atención de la salud) para implementar las políticas públicas de salud necesarias y apropiadas; 3) ausencia de la identificación de problemas y establecimiento de prioridades; 4) interacción inadecuada entre investigadores, políticos (tomadores de decisiones en materia de salud pública) y agencias de financiamiento (11). Todo lo anterior puede conducir a una incorrecta investigación traslacional (translational research) del escaso y cuestionable conocimiento generado en los países del Sur, lo cual puede resultar en la implementación de acciones que no son de impacto benéfico para las poblaciones afectadas.

En un trabajo reciente sobre cooperación internacional para el desarrollo (CID), Ripoll y Gothme (12) ponen en duda la posible utilidad de la CID como herramienta para el desarrollo. Para estos autores, existe una clara diferencia entre cooperación y ayuda y, como puntualizan, el predominio de la última en el panorama mundial da lugar a que la CID sea "una herramienta para el atraso", pues es unilateral y vela solo por los intereses del país "donante" o del Norte.

Ante este panorama se han propuesto diversas intervenciones por organismos internacionales tales como el fortalecimiento de la capacidad de realizar investigación (13), la necesidad de realizar investigación nacional "esencial" (14) y el planteamiento de los Objetivos de Desarrollo del Milenio (15), por citar las que se esperaba fueran de alto impacto. No obstante, se reconoce que, a pesar de los esfuerzos realizados a nivel mundial, las denominadas iniciativas globales en salud y los objetivos y metas propuestos no han sido alcanzados. Una de las posibles razones que puede aducirse al intentar entender este "fracaso" es que las intervenciones han estado enfocadas de manera primordial a la atención e investigación para la salud. Es necesario ser conscientes de que la salud es un estado resultante de la interacción de múltiples factores subyacentes y determinantes. Mientras no se atiendan, las desigualdades sociales, económicas, culturales y políticas, al mismo tiempo que se aborda el problema de salud, difícilmente se logrará un cambio y persistirán los problemas. Este es un reto mayúsculo sobre el cual es necesario trabajar efectivamente en colaboración a nivel internacional y nacional. 


\section{Colonialismo}

La historia de la humanidad se ha caracterizado a través de los siglos por el imperialismo dominante, es decir, la relación entre países basada en ideas de superioridad desde las cuales los países más poderosos aplican prácticas de dominación. Primero, el descubrimiento de América dio lugar a la creación de colonias en el nuevo continente y por tanto a relaciones asimétricas de poder. Segundo, la denominada era del imperialismo (1871-1914), relacionada con la creación de imperios coloniales por el reparto de África, dio lugar al llamado colonialismo (16).

La investigación para la salud no es ajena al colonialismo. Al igual que la economía mundial, que ha entrado en la era de la globalización, gran parte de la investigación biomédica se viene realizando en países del Sur, tendencia que continuará como todo parece indicar (17). Esto ha dado lugar a que los críticos de la investigación colaborativa Norte-Sur para la salud consideren este tipo de investigación, no sin razón, de carácter colonialista, neocolonialista, imperialista occidental o paternalista (18-22). Entre los argumentos aducidos se encuentra que el financiamiento por lo general proviene de los países del Norte; es común que estos últimos elijan los problemas de estudio, establezcan las prioridades y con frecuencia diseñen el proyecto, definan la metodología, de forma tal que los proyectos son "dictados" a los participantes de los países del Sur. Para ello recurren a la ya mencionada desigualdad en términos de carencia o deficiencias del equipo de trabajo, la infraestructura física, recursos administrativos; todo esto permite que con el discurso de "fortalecimiento de la capacidad" se disfrace el colonialismo-paternalismo hacia los países del Sur y se realicen proyectos que en ocasiones son de poca utilidad y beneficio para los afectados en los países del hemisferio más pobre.

En la interacción Norte-Sur han existido acusaciones de robo y fraude (23), de violación de los lineamientos internacionales (24), de renuencia de las organizaciones internacionales para atender los intereses e inquietudes de los países del Sur, así como una injusta distribución de los beneficios
(25) y, como ya se ha mencionado, de pobre participación de los investigadores de los países del Sur en las publicaciones (26). Todas estas prácticas han contribuido lamentablemente a una pérdida de confianza en la medida en que las investigaciones son dirigidas, desarrolladas y controladas desde países del Norte, sin atender las necesidades de los países del Sur.

Aunado a todo lo anterior, uno de los efectos negativos de la investigación en colaboración Norte-Sur es la competencia entre los escasos investigadores calificados de los países del Sur por obtener financiamiento. Es común que dichos investigadores se consideren afortunados de poder ser parte de una publicación internacional como colaboradores "invitados", situación que suele propiciar la desatención de problemas esenciales a nivel nacional (27).

Por otra parte, uno de los problemas que se viene agravando con el desarrollo científico y tecnológico es la utilización de materiales biológicos humanos, tema que preocupa a la investigación biomédica ligada a las grandes corporaciones de la industria farmacéutica por ser altamente lucrativa al permitir descubrimientos y patentes, los cuales con frecuencia no son aplicables o están fuera del alcance de la población de países del Sur. Si bien el problema de los ensayos clínicos para lograr la aprobación de nuevos medicamentos por parte de organismos reguladores ha sido ampliamente revisado y documentado en la literatura (28), las prioridades de investigación y el desarrollo de nuevos medicamentos están condicionados por el mercado. Para los hombres de negocios, una "buena" medicina es la que está desarrollada para una indicación redituable económicamente. Esto ha dado lugar a que las enfermedades infecciosas afecten más a los países en condiciones de pobreza: al perjudicar a una población que no permiten lucrarse, no reciben atención ni apoyo para el desarrollo de nuevos medicamentos.

\section{Explotación}

Dadas las condiciones de asimetría y colonialismo, y como consecuencia del imperialismo científico, surgen malas prácticas, comúnmente utilizadas dentro de la investigación colaborativa Norte-Sur 
para la salud; estas últimas dan lugar a la explotación, es decir, permiten tomar ventaja de la situación de desventaja en beneficio del más poderoso. Aunado a lo anterior, es habitual que las poblaciones de estudio de los países del Sur se encuentren en situación de vulnerabilidad, lo que favorece y permite con frecuencia las denominadas investigaciones "mosquito", "safari", "helicóptero", que consisten en llegar a una comunidad, extraer material, salir y publicar, sin pensar en el beneficio de la institución colaboradora (29). Por consiguiente, no hay formación de recursos humanos, transferencia de tecnología y mucho menos beneficio alguno para los afectados por el problema que se está estudiando, quienes radicados en comunidades afectadas viven en la pobreza. Es urgente eliminar la práctica de esta conducta inapropiada y asegurar una relación respetuosa y equitativa. En esencia, se requiere de mutua comprensión, voluntad y compromiso a largo plazo con el fin de evitar que la explotación de los países del Sur continúe.

Recientemente, con el apoyo de la Comisión Europea, se publicó en línea un excelente libro de casos de estudio sobre explotación de países del Sur con el fin de concienciar sobre este grave problema (30). Llama la atención que, en pleno tercer milenio, los casos de explotación en la investigación colaborativa Norte-Sur para la salud sigan ocurriendo a pesar de disponer de las declaraciones internacionales de ética de la investigación, incluyendo la Declaración Universal de Bioética y Derechos Humanos de la Unesco (31).

\section{Información}

El intercambio de información entre el Norte y el Sur es fundamental para el desarrollo de los sistemas de salud. Sin embargo, existe una serie de situaciones que han dificultado el hacer visible la información proveniente de países del Sur.

Podemos mencionar el hecho reconocido de que los editores de revistas biomédicas angloparlantes han prestado poca atención a las dificultades que enfrentan los investigadores de países del Sur para publicar. Debe recalcarse que el flujo de información entre el Norte y Sur debería ser de dos vías y que los editores de las compañías internacionales deberían comprometerse apoyando esta responsabilidad dual. Es una realidad lamentable que gran parte de la información de los países del Sur no es debidamente difundida por no considerarse prioritaria. El debido intercambio de información permitiría la atención de las cuestiones de salud desatendidas que afectan a países del Sur sumidos generalmente en condiciones de pobreza.

Se ha de subrayar, también, que mucha de la información que aparece en las publicaciones internacionales proviene de los países del Sur, que con frecuencia resultan ser "proveedores de datos", ya que los estudios se realizan en sus países. Así también, se sabe del "temor" de los investigadores del Sur a publicar por razones tales como: 1) problemas de dominio del lenguaje (generalmente inglés); 2) pobre acceso a la literatura actualizada en aspectos de salud; 3) no contar con una masa crítica a nivel local, regional o nacional a la cual acudir en busca de apoyo y asesoría; 3) muy poca comprensión y falta de apoyo por parte de los editores de revistas; 4) poco interés en información proveniente de los países pobres por parte de las editoras; 5) las revisiones no consideran las condiciones de los investigadores del Sur; 6) el denominado factor de impacto prioriza la investigación de los países del Norte (32).

Con todo, resulta esencial la creación de una relación de solidaridad internacional entre las comunidades científicas y entre las naciones para lograr avanzar en la realización de una investigación en colaboración que conduzca a un desarrollo humano global sostenible. Existe en la comunidad científica internacional un llamado urgente sobre la indispensable disponibilidad a nivel global de información científica sin costo.

\section{Comités de ética en investigación en salud}

Otra característica común en los países del Sur, que es todo un reto, es la desatención a la ética-bioética por parte de las autoridades estatales responsables. Ciertamente, en los documentos oficiales a nivel internacional es usual ver que se reporta la existencia de Comisiones Nacionales de Bioética (CNB), Comités de Ética de la Investigación en 
Salud (CEIs), etc., pero, como advierte Habermas (33), esto es habitual en las "democracias de fachada" en las cuales la simulación es una característica y por tanto otro reto más que abordar.

Las CNB no deben ser oficinas para el registro de CEIS, sino organismos responsables de su debida acreditación y seguimiento. En este punto es necesario trabajar intensamente e incluso elaborar guías de carácter internacional para la realización de esta actividad.

Los CEIs tienen responsabilidades bien definidas:

1) evaluar de manera ética (en todo lo amplio que esto significa) de los protocolos de investigación, teniendo particular cuidado cuando se trata de proyectos de investigación en colaboración Norte-Sur;

2) construir una cultura ética-bioética en sus instituciones, actividad dirigida a todos los involucrados en la investigación (desde autoridades hasta técnicos, estudiantes, personal de apoyo en estudios de campo) y a la comunidad afectada, para lograr la necesaria comprensión y, más que nada, valoración de la importancia de la ética en el quehacer de la investigación, y

3) monitorear y auditar los proyectos de investigación para asegurar y promover una conducta responsable (responsible conduct research), así como también prevenir y evitar conductas inapropiadas (misconduct research).

Los CEIs tienen además entre sus responsabilidades en relación con la investigación en colaboración Norte-Sur la protección de los participantes, sobre todo cuando se trata poblaciones en situación de vulnerabilidad, lo cual es común en este tipo de investigación. Para poder cumplir con ello, es imprescindible que: 1) cuenten con la capacitación apropiada, los recursos necesarios y la independencia para afrontar este reto; 2) sean capaces de reconocer cuestionamientos éticos culturalmente sensibles en entornos complejos, y 3) dispongan de un mecanismo de cumplimiento muy bien establecido (30).

Otra de las condiciones para poder cumplir con sus responsabilidades es una idónea conformación de los CEI. Con el paso de los años, la conformación se ha venido modificando en respuesta a los dilemas éticos que plantean los proyectos de investigación conforme avanza el desarrollo científico y tecnológico.

En la actualidad, con base en la importancia de los determinantes sociales de la salud y el valor social del conocimiento generado, existe la necesidad de que en los CEI, por su carácter multidisciplinario e interdisciplinario, se integren, además del personal calificado en ciencias de la salud, representantes de ciencias sociales (sociólogos, antropólogos), filósofos, bioeticistas, con la finalidad de realizar una revisión ética interdisciplinaria y por tanto integral. La revisión ética no debe de reducirse solamente a una tarea burocrática de chequeo de formatos (lista de cotejo o check list), que en los últimos años es un fenómeno frecuente.

En África usualmente no existen condiciones para que los CeIs sean capaces de cumplir sus funciones, debido a un completo listado de limitaciones: 1) insuficiente capacitación; 2) falta de recursos para estar actualizados; 3) presión por parte de los investigadores para recibir el dictamen de aprobación; 4) escasa participación o falta de continuidad de los integrantes del CEIS; 5) pobre reconocimiento de la importancia de los CEIS en su institución; 6) la falta consecuente de apoyo institucional; 7) no disponer de la autonomía e independencia para realizar sus labores, y 8) presión de los organismos financiadores, lo cual puede inducir un trato desigual de los protocolos en revisión (34). Estas limitantes y retos no son exclusivos de los países africanos, sino que son comunes en otros países del Sur.

De acuerdo con los lineamientos internacionales, está bien establecido y visto que todo proyecto de investigación en salud debe ser revisado y aprobado por un comité de ética independiente antes de iniciarse. Actualmente, se considera un requisito el que los proyectos sean revisados y aprobados por los CEIs de las instituciones del Norte y Sur participantes. Así también, según los estudios multicéntricos que involucran varios países del Sur, es altamente recomendable que se establezca una relación estrecha y que los CEIs compartan información sobre sus dictámenes. Esta medida favorecería el fortalecimiento de los cEIs del Sur y, a su vez, la urgente regulación de los del Norte. 


\section{Consideraciones bioéticas}

La literatura revisada con respecto a los desafíos y ética de la investigación colaborativa Norte-Sur para la salud presenta evidentemente un marcado predominio de publicaciones de autores de países del Norte. Esto aplica igualmente a los documentos y declaraciones internacionales sobre ética de la investigación, en los cuales la contribución de participantes de países del Sur es reducida. Por lo anterior, no es de extrañar que el abordaje ético de la investigación colaborativa Norte-Sur para la salud se ha caracterizado por un enfoque principialista anglosajón, apegado a los cuatro principios de la ética biomédica de Beuchamp y Childress (35).

Los principales desafíos identificados a los que se enfrenta la investigación colaborativa Norte-Sur para la salud, asimetría, colonialismo, explotación, información, comités de ética en investigación para la salud, plantean, tal como ha sido señalado, múltiples cuestionamientos de carácter multi e interdisciplinario. Por ello, si se pretende realmente lograr un cambio que redunde en el beneficio de los marginados, discriminados, pobres, afectados por enfermedades de la pobreza y, por ende, de la humanidad como un todo, es necesario el abordaje bioético muy acorde a la compleja realidad.

Así tenemos que el desafío identificado como asimetría - sin lugar a dudas el reto mayor- es resultado de múltiples factores económicos, sociales, culturales, políticos, ambientales, por lo cual, para lograr "modificarla" o al menos disminuir la profunda brecha entre países del Norte y del Sur, no basta con el enfoque ético tradicional basado en la ética principialista ya mencionada. Ligados a lo anterior, el colonialismo y la subsecuente explotación persisten en nuestros días. El desequilibrio en la información amerita una serie de cambios en la conducta de investigadores del Norte y del Sur, y tal como ha sido revisado de las editoriales internacionales responsables de la revisión y publicación de los resultados obtenidos. Ante esta situación es necesario construir una bioética de la investigación colaborativa Norte-Sur para la salud.

Si bien el objetivo principal del presente trabajo no es realizar una revisión de las teorías éticas desarrolladas a la fecha, con base en lo expuesto anteriormente, se ha de proponer la inclusión de otros principios - además de los multicitados de la ética biomédica - con el fin de avanzar en la debida revisión de los proyectos de investigación para la salud.

Ante la grave situación que enfrentamos en la actualidad, particularmente en los referidos efectos del cambio climático, así como los dilemas que surgen por el acelerado desarrollo científico y tecnológico, el principio de responsabilidad de Jonas (36) propone un nuevo imperativo categórico: "Actúa de tal modo que los efectos de tu acción sean compatibles con la permanencia de una vida humana auténtica", lo cual expresado de manera negativa diría "No pongas en peligro la continuidad indefinida de la humanidad en la Tierra". Jonas analiza y critica las éticas tradicionales por ser estrictamente antropocéntricas. Así también, consciente del salvaje e incontrolado desarrollo tecnológico y científico, este teórico aporta la visión del posible impacto con "potencial escatológico" del desarrollo de la tecnología sobre el futuro de la humanidad y de la vida en el mundo.

En la actualidad, la comunidad científica y tecnológica, así como los organismos internacionales reguladores, enfrentan un gran reto debido a los avances tecnológicos que permiten la edición del genoma humano. Por tal motivo, se vienen organizando reuniones internacionales para abordar el asunto con responsabilidad, pensando en las generaciones futuras. Ciertamente, la edición del genoma humano permitirá grandes avances en lo referente a la salud y por ello será necesario rescribir muchos capítulos sobre las ciencias y la ética de la salud.

La responsabilidad para existir requiere que el sujeto responsable tenga consciencia de su capacidad o de su incapacidad de enfrentar y responder al problema que la vida le plantea. La responsabilidad (del latín responsum) es la capacidad de dar respuestas eficaces a los graves problemas que nos plantea la compleja realidad actual. Así, hacerse realmente responsables de los problemas que plantea la investigación colaborativa Norte-Sur para la salud es un deber de quienes toman decisiones en el mundo actual, de los responsables de la gobernanza de todos los países, de las autoridades 
responsables de la salud internacional y nacional, de los investigadores y de los bioeticistas.

Así también, el principio de solidaridad debe incluirse en el abordaje de la investigación para la salud con énfasis en la investigación colaborativa Norte-Sur. La solidaridad se considera la expresión social de la fraternidad humana en todos los campos de la convivencia, imprescindible para el desarrollo integral de las personas y la edificación del porvenir común de la humanidad (37). A propósito de este principio se ha de mencionar la importancia que se le viene dando recientemente en el campo de la ética de la investigación de la salud pública, que ha estado regida por tres principios de la ética anglosajona: el de beneficencia, en términos de procurar el mayor bien posible a la comunidad; el de no maleficencia, en lo referente a prevenir, impedir o minimizar cualquier daño que la intervención pudiera causar a la comunidad, y el de respeto de la autonomía, de acuerdo al cual los individuos y la comunidad deben dar su consentimiento para la correcta aplicación de políticas y acciones de salud. La justicia ha sido incluida como principio, en términos de justicia social, con el fin de evitar discriminaciones arbitrarias de individuos afectados por las políticas de salud y reducir las inequidades en salud.

Dawson y Jennings (38) enfatizan el creciente interés por las cuestiones éticas que plantea la salud pública. Sin embargo, es inusual encontrar alguna mención a la solidaridad en las pautas y lineamientos internacionales, con excepción del informe sobre ética de salud pública del Nuffield Council of Bioethics (39). Estos autores toman el "segundo nivel" de Prainsack y Bruyx (40), en el que la solidaridad es considerada "un compromiso colectivo para sufragar los costes de la salud pública con el fin de ayudar a otros con quienes se comparte una situación o causa común". Con base en dicha propuesta, señalan que la solidaridad requiere de una acción pública, un acto que en sí mismo connota estar junto al "otro", identificarse con él y su posición, impulsado por la compasión y la comprensión. La acción en sí está orientada hacia el mejoramiento o corrección de alguna desventaja o injusticia. Para dichos autores, la idea de que "la solidaridad es un concepto relacional" resulta central, significa ser y estar "junto al otro", estar "para él otro" y "por el otro". Además, precisan que esta idea no pertenece en cuanto valor a la ética del deber, nadie está obligado a ser solidario, sino que es un concepto constitutivo, parte de nuestra naturaleza en cuanto seres biológicos sociales. Por lo tanto, plantean que la solidaridad debe ser un principio rector en la ética de la salud pública. Ahora bien, la interrogante es ¿cómo lograr la inclusión de dichos principios en la deliberación y toma de decisiones que nos permitan alcanzar consensos a nivel global?

Hemos señalado el marcado predominio en las publicaciones y documentos internacionales (pautas, lineamientos, guías) de organismos, instituciones, investigadores y tomadores de decisiones procedentes de países del Norte. Los "representantes" de los países del Sur con frecuencia no son dominantes, ya que es común la práctica de "designarlos" y no elegir de manera aristotélica al más capaz, al mejor preparado, al más consciente, responsable y humano. Esto amerita un cambio que les corresponde a los países del Sur.

Ante el sombrío panorama presentado, consideramos que la ética del discurso propuesta por los filósofos alemanes Apel y Habermas (41) es una herramienta que ayudaría en mucho a lograr alcanzar la meta propuesta. Si bien existe una corriente en Latinoamérica $(42,43)$ que ha cuestionado la ética discursiva, la misma ha dado lugar al establecimiento de un diálogo muy enriquecedor, particularmente entre Dussel y Apel (44).

Según Apel (45), los dos componentes constitutivos de la ética del discurso son, primero, el "discurso argumentativo" como medio para la fundamentación de las normas morales y del derecho de consenso. Se plantea entonces la necesidad de asumir una responsabilidad solidaria ante las consecuencias globales (primarias y secundarias) de la actividad humana colectiva, la cual, a su vez, debe ser entendida como una organización de esta responsabilidad en términos de praxis colectiva (corresponsabilidad). Así, esta ética se presenta como una vía de solución para la consolidación de una ética de la responsabilidad, entendiendo a esta como "la cooperación solidaria de los individuos ya en la fundamentación de normas morales y del 
derecho susceptible de consenso por medio del discurso argumentativo".

El segundo componente de esta ética es el "discurso argumentativo" como continente del a priori racional de la fundamentación del principio de la ética. Este último debe hacer posible una "fundamentación última del principio ético a partir del cual han de derivarse siempre todos los discursos argumentativos como discursos prácticos de la fundamentación de normas". Lo que se busca a través de los "discursos prácticos" es la susceptibilidad de consenso con pretensión de encontrar soluciones prácticas como respuesta a conflictos para todos los afectados.

De manera complementaria, Habermas (46) añade al "discurso argumentativo" la necesaria "acción comunicativa", pues considera que el éxito en el intento de fundamentar se obtiene si se identifica correctamente una pretensión de validez especial unida a los mandatos y a las normas, lo cual además debe darse en el contexto en que surgen los dilemas morales. Estas pretensiones deberán exponerse antes de todo análisis en los contextos de la "acción comunicativa". Con comunicativas se refiere a las interacciones en las cuales los participantes coordinan de común acuerdo simbólicamente sus planes de acción.

Ciertamente será necesario mucho esfuerzo y trabajo en conjunto, colaboración, cooperación, de manera consciente, responsable, solidaria, para en un futuro lograr modificar en principio el estatus que aún priva en el mundo actual de marcadas diferencias entre los países del Norte y del Sur.

\section{Señalamientos finales}

Sin lugar a dudas hay numerosas razones por las cuales es deseable que los investigadores sigan los estándares y pautas éticas internacionales establecidas. David y Resnik (47) han realizado una muy buena síntesis sobre la importancia de la ética en la investigación enfatizando en su contribución que se debe promover: 1) alcanzar los objetivos de la investigación (generación de conocimiento confiable) con apego al rigor científico evitando por tanto errores y conducta inapropiada; 2) los valores esenciales de confianza, respeto mutuo, responsabilidad, equidad e imparcialidad para trabajar en colaboración ; 3) la responsabilidad pública (transparencia, rendición de cuentas), dado que es muy importante considerando que la investigación es financiada; 4) la creación de un círculo virtuoso en la investigación logrando el apoyo de la comunidad, y 5) valores morales y sociales: responsabilidad social, respeto de la dignidad y los derechos humanos.

En el caso de las investigaciones para la salud en colaboración Norte-Sur, es de suma importancia que los investigadores de los países del Norte tomen consciencia de su situación privilegiada al trabajar en países con condiciones de salud muy por encima de las de los países del Sur y, de manera responsable, consideren la situación de los investigadores y población de los países del Sur para evitar cualquier riesgo de explotación.

Los datos compartidos deben maximizar los beneficios en salud (público e individual) y asegurar que la investigación sirva para fines de valor social. Para lograrlo es muy importante asegurar la calidad y comprensión de los datos compartidos, la validez científica y el valor social de los proyectos. Así mismo, los datos deben ser accesibles, permitir su uso de manera eficiente, así como su operatividad por los grupos colaboradores, y ser sostenibles, es decir, que el uso a futuro sea posible. El fundamento de todo lo anterior es el deber de compartir datos científicos y el derecho a la ciencia, que de manera kantiana debería de ser una norma universal.

Llama la atención un artículo reciente en el que se revisan los principios y normas relevantes para compartir responsablemente los datos de la investigación en salud a nivel internacional en la Unión Europea (48). Este referente es un claro ejemplo de investigación en colaboración Norte-Norte, entre países de la Unión Europea, en donde se tiene el cuidado de respetar principios y normas internacionales. Los principios identificados en un total de 31 artículos seleccionados revisados y las normas respectivas para promoverlos fueron agrupados en los siguientes rubros: 1) beneficio y valor social; 2) distribución equitativa de riesgos, beneficios y cargas; 3) respeto de los individuos y comunidades, y 4) confianza pública y compromiso. 
Al comparar el trabajo revisado sobre la ética de la investigación en colaboración Norte-Norte con la de la investigación en colaboración Norte-Sur, resulta evidente que hay diferencias notorias. Las características apuntadas en el presente trabajo, asimetría, intercambio de información, colonialismo, explotación, comités de ética de la investigación, para fines prácticos no existen, ya que los países participantes se encuentran en una situación y relación simétrica.

Esto nos lleva a señalar la posible existencia de un "doble estándar", o negligencia, por parte de algunos o todos los involucrados en las interacciones Norte-Sur. Lo anterior es muy lamentable si consideramos que existen lineamientos, pautas, principios y normas suficientes para conducir la investigación en colaboración Norte-Sur de manera ética. Una vez más es necesario reiterar que la ética es una ciencia práctica y por tanto es el resultado de acciones concretas. A nivel internacional es urgente pasar del discurso a las acciones que permitan realmente avanzar en la posible consecución de un mundo más justo en la distribución de bienes existentes, salud, y educación.

Tal como señala Boff (49), "estamos obligados a desarrollar un ethos de la responsabilidad ilimitada [...] es urgente pasar del eje de la razón calculadora al eje de la colaboración que utiliza la razón cordial".

En el mundo actual enfrentamos problemas muy graves como el pragmatismo avasallador, el terrorismo racial, los discursos y crímenes de odio, el resurgimiento del nazismo con la llamada "supremacía blanca" y una deshumanización con cada vez menor consciencia y responsabilidad social. Ante este panorama, proponer la igualdad entre países del Norte y del Sur es una utopía. Sin embargo, como bien dice Galeano, la utopía es como el horizonte, caminamos hacia él y se aleja, pero nos hace movernos y avanzar. Tal como dice Sontag en el cuento sobre la descendiente de Noé, el cual se cita al inicio de este trabajo, necesitamos creer que aun cuando: "Es cierto no se puede salvar el mundo ¿y si actuamos como si pudiera salvarse? [...] Sí — dijo el pájaro agorero más animado-... Casi es posible que se pueda salvar el mundo" (50). Por lo tanto, se propone la construcción de una bioética de la investigación para la salud en colaboración entre los países del Norte y del Sur con el fin de reducir el abismo existente entre los mismos.

\section{Referencias bibliográficas}

1. Binka F. Editorial: North-South research collaborations: a move towards a true partnership? Trop Med \& Int Health. 2005 febr. 23; 10(3):207-209.

2. The Third World Academy of Sciences (twas). 'The World Academy of Sciences for the advancement of science in developing countries', 2020. [en línea]. Disponible en: https://twas.org. [Citado el 08 de enero de 2020].

3. Nuyens Y. No Development Without Research. Global Forum for Health Research; 2005.

4. Asnáriz T. ¿De qué hablamos cuando hablamos de bioética? Selecciones de Bioética. 2010; 16:22-30. Disponible en: https://cenalbe.org.co/PDF/seleccionesNo.16.pdf

5. Paranagua de Santana J. Bioethical Dimensions of International Cooperation for Health: still a controversial issue? Cien Saude Colet. 2017 jul.;22(7):2245-2250. DOI: 10.1590/1413-81232017227.02822017.

6. Gaillard JF. North-South Research Partnership: Is Collaboration Possible between Unequal Partners? Knowledge \& Policy. 1994 jun.;7(2):31-63. DoI: 10.1007/ BF02692761

7. Maina-Ahlberg B, Nordberg E, Ran Tomson G. North-South Health Research Collaboration: Challenges in Institutional Interaction. Soc Sci Med. 1997 abr.;44(8):1229-38.

8. Commission on Health Research for Development (COHRED). Health Research: Essential Link to Equity in Development. Oxford University Press, Oxford; 1990.

9. Ehrenberg JP, Ault SK. Neglected diseases of neglected populations: Thinking to reshape the determinants of health in Latin America and the Caribbean. BMC Public Health [Internet]. 2005 nov.11;5(119). Disponible en: https://rdcu.be/b4aD9 doi:10.1186/1471-2458-5119.

10. Jentsch B, Pilley C. Research relationships between the South and the North: Cinderella and the ugly sisters? Soc Sci Med. 2003 nov.;57:1957-1967. doi:10.1016/ S0277-9536(03)00060-1.

11. Cash-Gibson L, Guerra G, Salgado-De-Snyder VN. SDH-NET: A South-North-South collaboration to build sustainable research capacities on social determinants of health in low- and middle-income 
countries. Health Res Policy Syst. 2015 oct. 12;13:45. DOI: 10.1186/s12961-015-0048-1.

12. Ripoll A, Gothme R. La cooperación internacional: herramienta de desarrollo o de atraso. Rev. Latinoam. bioét. 2014 dic. 11;15(1):54-63.

13. World Health Organization WHO. 'Strengthening research capacity', 2019. [en línea]. Disponible en: https://www.who.int/tdr/capacity/en/. [Citado el 08 de enero de 2020].

14. Council on Health Research for Development, editor. Essential National Health Research [Internet]. Disponible en: https: www.cohred.org/downloads/586.pdf

15. United Nations. 'Millennium Development Goals Reports', 2015. [en línea]. Naciones Unidas. Disponible en: http://www.un.org/millenniumgoals/ reports.shtml [Citado el 08 de enero de 2020].

16. Granados-Erazo O. Imperios y colonialismo, 18701914 ¿una era de globalización, geopolítica o nacionalismo económico? Relac Int Estrateg Segur. 2010 jun.;5(1):51-82.

17. Sathar A, Dhai A, Van Der Linde S. Collaborative international research: ethical and regulatory issues pertaining to human biological materials at a south african institutional research ethics committee. Dev World Bioeth. 2014 dic.;14(3):150-157. Doi:10.1111/ dewb.12018.

18. Trostle J. Research capacity building in international health: definitions, evaluations and strategies for success. Soc Sci Med. 1992 dic.; 35:1321-1324.

19. Hernandez-Aguado I. Neocolonialism and epidemiology. J Epidemiol Community Health. 2001 mar.;55(3):153. DoI: 10.1136/jech.55.3.153.

20. Braveman PA. Epidemiology and (neo-)colonialism. J Epidemiol Community Health. 2001 mar.55(3):160161.

21. Lang T. Public health and colonialism: a new or old problem? J Epidemiol Community Health. 2001 mar.; 55:162:163.

22. Benatar SR. Reflections and recommendations on research ethics in developing countries. Soc Sci Med. 2002;54(7):1131-1141. doi:10.1016/s02779536(01)00327-6.

23. Andanda PA. Human tissue related invention; ownership and intellectual property rights in international collaborative research in developing countries. J Med Ethics. 2008 mar.;34:171-179.

24. Mudur G. Indian scientists object to export of human biological material for research. BMJ. 2002 nov. 2;325(7371):990.
25. Emerson CI, Singer PA, Upshur RE. Access and use of human tissues from the developing world; ethical challenges and a way forward using a tissue trust. BMC Medical Ethics. 2011 en. 25;12:2-5. doi:10.1186/14726939-11-6.

26. Emmanuel EJ, Wendler D, Killen J, Grady C. What makes clinical research in Developing countries ethical? The benchmarks of ethical research. J Infect Dis. 2004 feb. 17;189(5):930-937. doi:10.1086/381709.

27. Trostle J, Simon J. Building applied health research capacity in less-developed countries: Problems encountered by the ADDR project. Soc Sci Med. 1992; 35(11),1379-1387.

28. Páez R. Pautas bioéticas. La industria farmacéutica entre la ciencia y el mercado. $2^{\text {a }}$ edición. México: fce; 2018, 335 p. isbn 978-607-16-5849.

29. Andrade-Narvaez FJ. Ética de las enfermedades tropicales desatendidas. Focus 48. Suiza: Globethcis.net; 2019. Cap. 4, Ética de la investigación en colaboración Norte-Sur; p. 77-92.

30. Schroeder D, Cook J, Hirsch F, Fenet S, Muthuswamy V. Ethics Dumping, Case Studies from North-South Research Collaborations. Suiza: Springer Open; 2018.

31. Páez-Moreno R. Justa distribución de beneficios en las declaraciones internacionales de ética de la investigación. Salud Pública de Méx. 2012 nov.-dic.;54(6):637-643.

32. Horton R. North and south: bridging the information gap. Lancet. 2000 jun. 24;355:2231-2236. doi:10.1016/ s0140-6736(00)02414-4.

33. Habermas J. La ética del discurso y la cuestión de la verdad. Chile: Escuela de Filosofía Universidad ARCIS; 2003. Disponible en: https://www.philosophia. cl/biblioteca/habermas/Habermas\%20-\%20La\%20 \%EF\%BF\%BDtica\%20del\%20discurso\%20y\%20la\%20 cuesti\%EF\%BF\%BDn\%20de\%20la\%20verdad.pdf.

34. Nyika A, Kilama W, Chilengi R, Tangwa G, Tindana P, Ndebele P., Ikingura J. Composition, training needs and independence of ethics review committees across Africa: are the gate-keepers rising to the emerging challenges? J Med Ethics. 2009 mar.;35(3):189-193.

35. Beauchamp T, Childress J. Principles of Biomedical Ethics. Nueva York: Oxford University Press; 1994.

36. Jonas H. El Principio de Responsabilidad: ensayo de una ética para la civilización tecnológica. Barcelona: Herder; 1995.

37. Suarez Hernández G. La solidaridad como principio de la bioética. En IX Congreso Internacional De La fibip y I Congreso Internacional De Bioética Del Centro 
Juan Pablo II, La Habana, mayo de 2013. Disponible en: http://www.cbioetica.org/revista/133/133-0409.pdf

38. Dawson A, Jennings B. The Place of Solidarity in Public Health Ethics. Public Health Reviews. 2013;34(1):65-79.

39. Public Health: Ethical Issues. Londres: Nuffield Council of Bioethics; 2007.

40. Prainsack B, Buyx A. Solidarity: Reflections on an Emerging Concept in Bioethics. Londres: Nuffield Council of Bioethics; 2011.

41. Rivas-García R. Aproximación de la "ética del discurso" de Apel y Habermas, como ética racional ante la crisis de la razón. En-clav. pen. 2007 jun.;1(1):63-89.

42. Michelini DJ, Malinaldi R, De Zan J. Ética del discurso. Recepción y críticas desde Latinoamérica. Argentina: Ediciones Del Icala; 2007.

43. Dussel E, Apel KO. Ética del discurso y ética de la liberación. Madrid: Trotta; 2004.

44. Apel KO. La ética del Discurso como ética de la responsabilidad: una transformación postmetafísica de la ética de Kant. En: Dussel E, Apel KO. Ética del discurso y ética de la liberación. Madrid: Trotta; 2004.

45. Apel KO. Ética del discurso, democracia y derecho de gentes. Invenio. 2006;9(17):19-33

46. Habermas J. Aclaraciones a la ética del discurso. Madrid: Trota; 2000.

47. David B, Resnik JD. What is ethics in research and why is it important? 2015; National Institute of Environmental Health Sciences. Disponible en: http://www. niehs.nih.gov/research/ resources/bioethics/whatis/

48. Kalkman S, Mostert M, Gerlinger C, Van Delden JJM, Van Thiel GJMW. Responsible data sharing in international health research: a systematic review of principles and norms. BMC Medical Ethics. 2019 mar. 28;20:21 https://doi.org/10.1186/s12910-019-0359-9.

49. Boff L. Ética y Moral. La búsqueda de los fundamentos. España: Sal Terrae; 2004.

50. Sontag S. Declaración, Cuentos reunidos. España: Literatura Random House; 2018. 